\title{
Trial Disease Assessments Domain
}

National Cancer Institute

\section{Source}

National Cancer Institute. Trial Disease Assessments Domain. NCI Thesaurus. Code C117699.

A subject domain utilized for the submission of information encompassing and representing data, vocabulary or records related to trial disease assessments. 